\title{
Tratamiento electroquímico de aguas que contienen antibióticos $\beta$-lactámicos
}

\author{
Electrochemical Treatment of Water Polluted with $\beta$-Lactam Antibiotics
}

\author{
A. L. Giraldo Aguirre ${ }^{\mathrm{a}}$ \\ E. D. Erazo Erazo ${ }^{\mathrm{a}}$ \\ O. A. Flórez Acosta ${ }^{a}$ \\ E. A. Serna Galvis ${ }^{b}$ \\ R. A. Torres Palma ${ }^{b, *}$
}

Recepción: 30-ago-2015

Aceptación: 20-ene-2016

\begin{abstract}
Resumen
Oxacilina (OXA), Cloxacilina (CLX) y Dicloxacilina (DCX) son antibióticos $\beta$-lactámicos usados para tratar infecciones producidas por microorganismos grampositivos y gramnegativos; estas sustancias pueden alcanzar los sistemas acuáticos debido a su inadecuada disposición o a deficiencias de los sistemas de tratamiento de aguas, originando un serio riesgo de proliferación en el ambiente de bacterias resistentes a estos antibióticos. El presente trabajo investigó la degradación de OXA, CLX y DCX mediante oxidación anódica, empleando un ánodo de $\mathrm{Ti} / \mathrm{IrO}_{2}$. Un diseño experimental, evaluando las variables 'corriente aplicada', 'concentración de $\mathrm{NaCl}$ ' y 'concentración de sustancia', permitió determinar las condiciones más favorables para la degradación de los $\beta$-lactámicos ensayados. Las mejores eficiencias, en términos de la remoción del contaminante y su actividad antibiótica, fueron obtenidas a altos niveles de corriente aplicada (121 mA) y concentración media de $\mathrm{NaCl}\left(0,225 \mathrm{~mol} \mathrm{~L}^{-1}\right)$ como electrolito soporte; mientras que la concentración del antibiótico no ejerció un efecto significativo sobre el desempeño del sistema. Los antibióticos OXA, CLX y DCX (203 $\left.\mu \mathrm{mol} \mathrm{L}{ }^{-1}\right)$ experimentaron, en forma independiente, una completa remoción a los 5 min de tratamiento, por una vía de degradación mediada por especies reactivas de cloro electrogeneradas en la superficie del ánodo. De manera interesante, se observó una pérdida completa de la actividad antimicrobiana tan pronto como desapareció el compuesto padre, indicando que los productos de degradación no presentan potencia antibiótica, inclusive algunos de estos productos formados inicialmente son también degradados por el sistema electroquímico. Los resultados indicaron que la oxidación electroquímica tiene gran proyección para el tratamiento de aguas que contienen antibióticos $\beta$-lactámicos, debido a su alta eficiencia tanto para remover el contaminante como para eliminar la actividad antimicrobiana de estos compuestos en periodos cortos de tiempo.
\end{abstract}

Palabras clave: Isoxazolilpenicilinas, oxidación anódica, actividad antimicrobiana, ánodo tipo DSA.

\footnotetext{
${ }^{a}$ Departamento de Farmacia, Facultad de Ciencias Farmacéuticas y Alimentarias, Universidad de Antioquia, Colombia.

*Autor de correspondencia: ricardo.torres@udea.edu.co

${ }^{b}$ Grupo de Investigación en Remediación Ambiental y Biocatálisis (GIRAB), Instituto de Química, Facultad de Ciencias Exactas y Naturales, Universidad de Antioquia, UdeA, Colombia.
} 


\begin{abstract}
Oxacillin (OXA), cloxacillin (CLX) and Dicloxacillin (DCX) are $\beta$-lactam antibiotics, used to treat infections caused by gram-positive and gram-negative microorganisms, which can reach aquatic systems due to improper disposal or deficient water system treatments, thus representing a risk to the environment. With this work, the degradation of these $\beta$-lactam antibiotics was investigated by anodic oxidation using a DSA anode $\mathrm{Ti} / \mathrm{IrO}_{2}$. An experimental design with variables assessing current density, supporting electrolyte, and the concentration of substance, allowed to evaluate the most favorable conditions for the degradation of the $\beta$-lactams tested. The best efficiencies in terms of pollutant and antibiotic activity removals, were obtained at high current level $(121 \mathrm{~mA})$ and average concentration of $\mathrm{NaCl}\left(0.225 \mathrm{~mol} \mathrm{~L}^{-1}\right)$ as supporting electrolyte; while the concentration of the antibiotic did not exert a significant effect on the system performance. Under these conditions, the antibiotics OXA, CLX and DCX $(203 \mu \mathrm{mol} \mathrm{L}-1)$, independently experienced a complete removal after $5 \mathrm{~min}$ of treatment, by a degradation pathway, mediated by the action of reactive chlorine species, electrogenerated on the anode surface. Relevant is, that a complete loss of antimicrobial activity was observed, as soon as the parent compound disappeared, indicating that the degradation products do not have an antibiotic potency. Even some by-products were also eliminated by the electrochemical action. The electrochemical oxidation is a technology that has great projection for the treatment of water containing $\beta$-lactam antibiotics, due to its high efficiency for both the removal of antibiotics and their antimicrobial activity in short periods of time.
\end{abstract}

Key words: Isoxazolyl Penicillins, Anodic Oxidation, Antimicrobial Activity, Anode Type-DSA.

\section{Introducción}

Desde su descubrimiento y su introducción como fármacos, hace unos ochenta y cinco años, los agentes quimioterapéuticos antimicrobianos naturales, como la penicilina; semisintéticos, como las cefalosporinas, y sintéticos, como las sulfonamidas y las quinolonas, han jugado un papel esencial en la disminución de la morbilidad y la mortalidad causada por las enfermedades infecciosas. Sin embargo, su amplia utilización, incluyendo el mal uso y el abuso, no solamente en el tratamiento y la prevención de infecciones microbianas en humanos y animales, sino también como promotores de crecimiento en la producción animal y en la agricultura, ha hecho que la presencia de antibióticos en el ambiente represente un riesgo para la salud de los seres vivos [1-3].

La presencia de antibióticos en el ambiente genera en la actualidad problemas de toxicidad, alergia [4,5] y resistencia bacteriana [2]. La resistencia a los antibióticos por parte de los microorganismos es un fenómeno grave y creciente en la medicina contemporánea, al punto de ser considerado uno de los problemas de salud pública más eminentes del siglo XXI.

Aunque la evolución de las cepas resistentes es un fenómeno natural que ocurre cuando los microorganismos se ven expuestos a fármacos antimicrobia- nos, y es posible un intercambio de características de resistencia entre ciertos tipos de bacterias, el uso inapropiado de estos medicamentos, las prácticas inadecuadas para el control de las infecciones y la presencia de antibióticos en el ambiente propician la propagación de la resistencia a los antimicrobianos y aceleran este fenómeno natural [6].

Un caso muy difundido de generación de resistencia se presenta con los antibióticos $\beta$-lactámicos, que incluyen derivados de la penicilina, las cefalosporinas, las carbapenemas, los monobactamicos, los carbacefem y los inhibidores de la betalactamasa ( $\beta$-lactamasa). Como ejemplos típicos, se conocen casos de Staphylococcus aureus meticilin-resistentes o Streptococcus pneumoniae penicilin-resistentes [7]. Con el fin de prevenir estos procesos de aceleración de la transferencia de resistencia a los antimicrobianos surge la necesidad de intervenir en los procesos de disposición sus residuos.

La OXA, CLX y DCX (figura 1) son antibióticos semisintéticos, miembros del grupo de antibióticos $\beta$-lactámicos resistentes a penicilinasa, conocidas como isoxazolilpenicilinas, de gran consumo en Colombia para el tratamiento de infecciones de la piel y la estructura de la piel, ocasionadas por cocos aerobios grampositivos, y como terapia de seguimiento después del tratamiento intravenoso para 
la osteomielitis $[9,10]$. Un alto porcentaje de los antibióticos consumidos por los seres humanos y animales en los hospitales o por prescripción, se excreta sin cambios a través de la orina y las heces en las aguas residuales domésticas, y son llevados a las plantas de tratamiento de aguas residuales (PTAR). En estas plantas dichos compuestos son eliminados solo parcialmente y buena parte de ellos y sus derivados son liberados al ambiente a través de los efluentes [8]. Por lo tanto, se hace urgente desarrollar sistemas de tratamiento eficientes que permitan tanto la eliminación de los antibióticos como de la actividad antimicrobiana de las aguas tratadas.

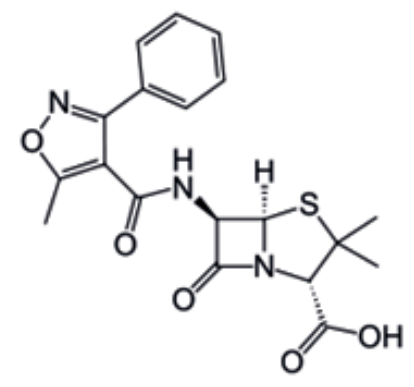

(a)

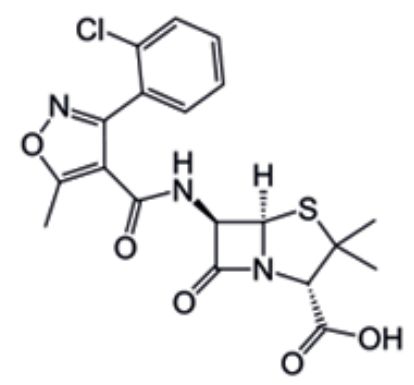

(b)

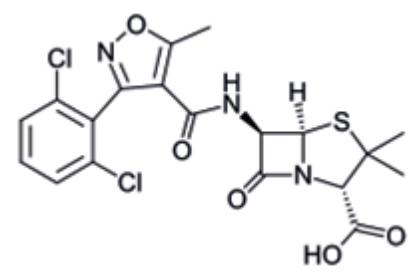

(c)

Figura 1. Estructura química de la (a) OXA, (b) CLX y (c) DCX.

En la última década, con el desarrollo de electrodos altamente eficientes y relativamente económicos, como los ánodos dimensionalmente estables (DSA), el uso de la oxidación electroquímica como alternativa al tratamiento de aguas que contienen contaminantes orgánicos ha despertado gran interés en la comunidad científica. Dentro de las ventajas que presenta esta tecnología cabe destacar su fácil automatización y el hecho de ser una metodología ambientalmente amigable, pues el reactivo principal es el electrón [11].

La oxidación electroquímica se basa en la aplicación de una corriente eléctrica o una diferencia de potencial entre dos electrodos (ánodo y cátodo), con lo cual se pueden generar radicales hidroxilo $\mathrm{u}$ otras especies oxidantes, dependiendo del material del ánodo empleado y del tipo de electrolito de soporte utilizado. De esta manera, la electrooxidación puede ser directa o indirecta; en el primer caso, el contaminante se degradada en el ánodo mediante una reacción de transferencia de carga, y en el segundo, el contaminante se elimina gracias a la acción de especies formadas durante la oxidación del agua o electrolitos presentes en la solución [12-16].

Con este trabajo se pretende evaluar la degradación de antibióticos $\beta$-lactámicos tipo isoxazolilpenicilinas en aguas, mediante la oxidación anódica con $\mathrm{Ti} / \mathrm{IrO}_{2}$, el ánodo tipo DSA más comúnmente utilizado. Mediante un diseño experimental se determina el efecto de tres variables sobre el desempeño cinético óptimo del proceso, utilizando la CLX como modelo. La eficiencia del proceso en términos de la eliminación del sustrato inicial, de la pérdida de la actividad antimicrobiana y de la acumulación de sustancias oxidantes es también evaluada. Finalmente, se estudia el seguimiento a la aparición y desaparición de los subproductos de degradación formados durante una hora de reacción.

\section{Materiales y métodos}

\section{Reactivos y sistema electroquímico}

La OXA, con pureza del 95\%, se obtuvo de Sigma Aldrich ${ }^{\circledR}$. La CLX y la DCX, materias primas, con una pureza $98,2 \%$, fueron donadas por Syntofarma S. A. (Colombia). El Cloruro de sodio y el yoduro de potasio se obtuvieron de Carlo Erba ${ }^{\circledR}$, y los solventes acetonitrilo, metanol y el heptamolibdato de amonio fueron marca Merck ${ }^{\circledR}$.

Las soluciones de los antibióticos se prepararon disolviendo la cantidad correspondiente del reactivo en agua desionizada. La medida del $\mathrm{pH}$ fue realizada usando un $\mathrm{pH}$-metro marca Jenway ${ }^{\circledR}$. El sistema 
electroquímico operó con un ánodo de $\mathrm{Ti} / \mathrm{IrO}_{2}$ (4 $\mathrm{cm}^{2}$ ) y un cátodo en espiral de zirconio de $10 \mathrm{~cm}$, usando $150 \mathrm{~mL}$ de solución.

\section{Análisis de isozaxolilpenicilinas (OXA, CLX y DCX)}

La cuantificación de cada antibiótico $\beta$-lactámico se realizó por medio de un HPLC marca Waters con un detector UV $(\lambda=225 \mathrm{~nm})$ y una columna RP-18 (LichrosPher ${ }^{\circledR}, 250$ x 4,6 mm y DI $5 \mu \mathrm{m}$ ). La separación óptima se llevó a cabo empleando una mezcla buffer fosfato:acetonitrilo:metanol (64:27:9) [17], a un flujo de $0,6 \mathrm{~mL} \mathrm{~min}^{-1} \mathrm{y}$ un volumen de inyección de $20 \mu \mathrm{L}$. La determinación de las sustancias oxidantes electrogeneradas se realizó por el método yodométrico, usando un espectrofotómetro Shimadzu UV-1700. La potencia antibiótica se determinó mediante el método de difusión en placa [17].

\section{Resultados y discusión}

\section{Optimización de las condiciones de análisis}

En la figura 2 se presenta el cromatograma de la separación de los tres antibióticos empleando el método propuesto en la farmacopea USP 35/NF 30, como se describe en la sección de materiales y métodos. Los tiempos de retención fueron cercanos a los 8,0 min, 10,0 min y 16,0 min para la OXA, la CLX y la DCX, respectivamente.

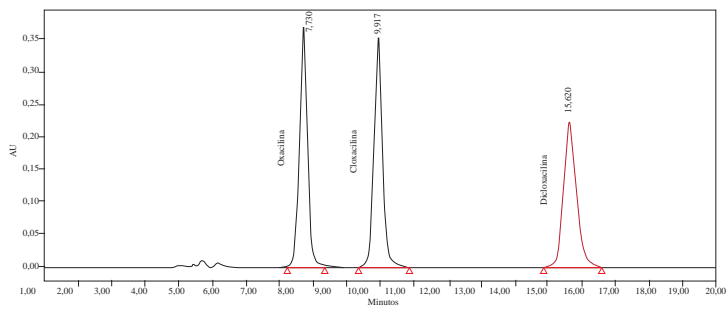

Figura 2. Cromatogramas obtenidos para las isoxazolilpenicilinas (Oxacilina, Cloxacilina y, Dicloxacilina).

El método cromatográfico mostró una buena separación, especificidad y sensibilidad, aun en presencia de los intermediarios de degradación. Se realizaron las respectivas curvas de calibración, en el rango de 3 a 100 ppm, para cada uno de los antibióticos, indicando coeficientes de correlación de 0,9974 para OXA, 0,9997 para CLX y 0,9992 para DCX, lo que refleja una buena relación lineal del método en el intervalo de concentraciones evaluado. La prueba de bondad de ajuste presentó valores superiores con respecto al valor $p 0,05$, indicando que las curvas de calibración desarrolladas son adecuadas para el seguimiento de los compuestos.

\section{Evaluación del efecto de parámetros y determi- nación de las condiciones de degradación de los antibióticos isoxazolilpenicilina}

Tomando la CLX como modelo de las isoxazolilpenicilinas, se realizó un diseño factorial $2^{K}$, incluyendo dos puntos centrales, para evaluar el efecto de la corriente aplicada entre 13 y $121 \mathrm{~mA}$, la concentración del antibiótico entre 6 y $1624 \mu \mathrm{mol} \mathrm{L}{ }^{-1}$ y el cloruro de sodio como electrolito soporte entre 0,05 y $0,4 \mathrm{~mol} \mathrm{~L}^{-1}$. Se efectuaron 10 ensayos por duplicado, empleando como variable de respuesta la velocidad de degradación durante los primeros 20 minutos, excepto para una concentración del contaminante de $6 \mu \mathrm{mol} \mathrm{L}^{-1}$, la cual, dada su rápida degradación, fue evaluada durante los primeros 30 segundos de reacción. En la tabla 1 se resume el diseño de experimentos empleado junto con la velocidad de degradación obtenida en cada caso.

La figura 3(a) muestra el efecto de los parámetros evaluados. La velocidad de degradación se incrementa con el aumento de la corriente y de la concentración de cloruro de sodio; en cambio, la concentración del contaminante no parece tener un efecto significativo en la eficiencia del proceso. De otra parte, además de las variables individuales, la interacción entre las variables estudiadas puede también afectar la velocidad de degradación del antibiótico. La figura 3(b) muestra la interacción entre las variables estudiadas; en ella, una mayor diferencia en las pendientes de las curvas indica mayor interacción entre las variables; de esta manera, una mayor interacción existe entre la corriente aplicada y la concentración de electrolito, al igual que entre las concentraciones de electrolito y sustrato.

Con el objeto de determinar cuáles de las variables estudiadas y de sus interacciones afectaron significativamente el proceso, la figura 4 muestra la expresión gráfica de la prueba ANOVA (diagrama de Pareto) [18]. Este grafico presenta tanto la magnitud como la importancia de los efectos y sus interacciones. El estudio fue realizado con un $95 \%$ de confianza. El diagrama de Pareto contiene una línea de referencia, y cualquier efecto superior a esta línea afecta de ma- 
A. L. Giraldo Aguirre et al.

Tabla 1. Diseño Experimental.

\begin{tabular}{ccccc}
\hline Bloque & $\begin{array}{c}\text { Concentración } \\
\text { sustancia } \\
\mu \text { mol L }\end{array}$ & $\begin{array}{c}\text { Corriente } \\
\text { aplicada } \\
\mathrm{mA}\end{array}$ & $\begin{array}{c}\text { Concentración } \\
\text { electrolito } \\
\text { soporte mol L }\end{array}$ & $\begin{array}{c}\text { Velocidad de } \\
\text { degradación } \\
\mu \text { mol L }^{-1} \mathrm{~s}^{-1}\end{array}$ \\
\hline 1 & 6 & 121 & 0,4 & 0,4562 \\
1 & 815 & 67 & 0,225 & 0,3433 \\
1 & 1624 & 13 & 0,4 & 0,1563 \\
1 & 6 & 13 & 0,05 & 0,0097 \\
1 & 815 & 67 & 0,225 & 0,2312 \\
1 & 1624 & 121 & 0,05 & 0,0288 \\
1 & 6 & 121 & 0,05 & 0,4355 \\
1 & 6 & 13 & 0,4 & 0,0382 \\
1 & 1624 & 13 & 0,05 & 0,0974 \\
1 & 1624 & 121 & 0,4 & 0,8237 \\
2 & 6 & 121 & 0,4 & 0,9770 \\
2 & 815 & 67 & 0,225 & 0,2783 \\
2 & 1624 & 13 & 0,4 & 0,1202 \\
2 & 6 & 13 & 0,05 & 0,005 \\
2 & 815 & 67 & 0,225 & 0,2767 \\
2 & 1624 & 121 & 0,05 & 0,032 \\
2 & 6 & 121 & 0,05 & 0,4309 \\
2 & 6 & 13 & 0,4 & 0,0558 \\
2 & 1624 & 13 & 0,05 & 0,0719 \\
2 & 1624 & 121 & 0,4 & 0,8422 \\
\hline & & & &
\end{tabular}

nera significativa el tratamiento electroquímico de los antibióticos [18-19].

A partir de estos resultados es posible concluir que la corriente aplicada, la concentración de electrolito de soporte, así como la interacción entre estos dos parámetros, fueron las variables con un impacto significativo en el sistema electroquímico. Como consecuencia, se establecieron como condiciones favorables para la degradación electroquímica de las isoxazolilpenicilinas una corriente de $121 \mathrm{~mA}$ y una concentración de $0,225 \mathrm{~mol} \mathrm{~L}^{-1}$ de $\mathrm{NaCl}$. Por otro lado, la concentración del compuesto no afecta el desempeño del sistema y, por lo tanto, en posteriores experimentos se empleó una concentración de $\approx 203$ $\mu \mathrm{mol} \mathrm{L}{ }^{-1}$ del antibiótico.

Determinación de la vía de degradación y características del tratamiento electroquímico de los antibióticos isoxazolilpenicilina

El resultado de someter soluciones acuosas de OXA, CLX y DCX $\left(203 \mu \mathrm{mol} \mathrm{L}{ }^{-1}\right)$ a electrooxidación, en forma independiente, se observa en la figura 5. En esta figura se muestra cómo en solo 4 minutos se reduce en más del $99 \%$ la concentración inicial de los fármacos, siguiendo una aparente cinética de orden cero. Es interesante anotar que, aunque el sistema remueve eficientemente los compuestos estudiados, la OXA, único antibiótico que no contiene cloro en su estructura (figura 1), se degrada más

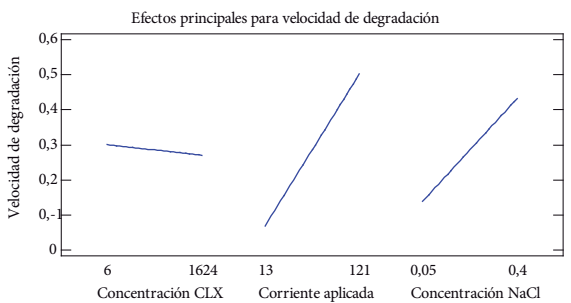

(a)

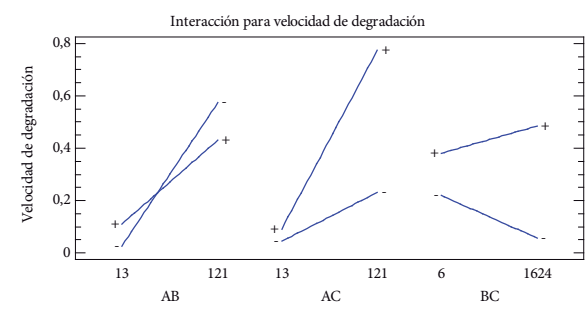

(b)

Figura 3. Gráficos de efectos principales (a) y gráfico de interacciones (b) obtenidos con el diseño experimental indicado en la tabla 1. (A: Corriente aplicada; B: Concentración del antibiótico; C: Concentración de $\mathrm{NaCl}$ ).

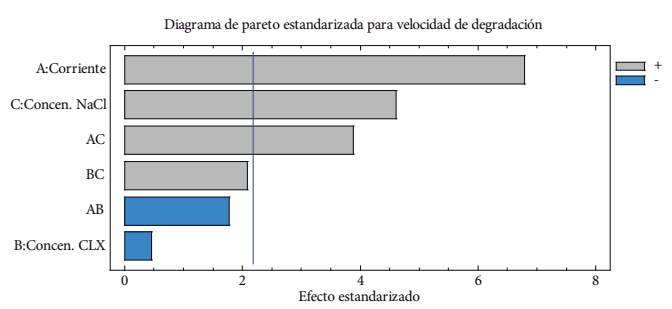

Figura 4. Diagrama de pareto estandarizado.

lentamente; dicho comportamiento sugiere el enlace cloro-carbono aromático como uno de los sitios posibles de reacción.

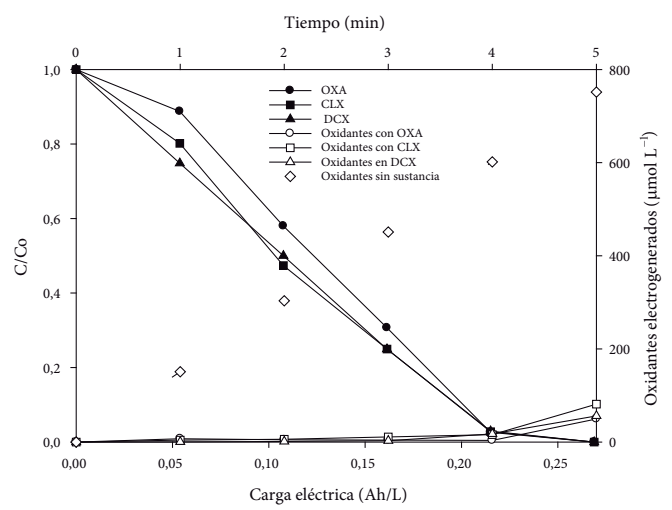

Figura 5. Degradación electroquímica de los antibióticos $\beta$-lactámicos isoxazolilpenicilinas $203 \mu \mathrm{mol} \mathrm{L}{ }^{-1}$, $121 \mathrm{~mA}, 0,225 \mathrm{~mol} \mathrm{~L}^{-1} \mathrm{NaCl}, 5$ minutos de tratamiento.

Con el objeto de entender la vía de degradación 
(directa o indirecta), en la figura 5 se muestra la acumulación de especies oxidantes en ausencia y en presencia de los contaminantes bajo estudio. En ausencia de las isozaxolilpenicilinas, se observa una acumulación progresiva de sustancias oxidantes conforme avanza el proceso. De hecho, en ausencia del antibiótico, luego de 5 minutos se tiene una concentración de $\sim 751 \mu \mathrm{mol} \mathrm{L} \mathrm{L}^{-1}$ de sustancias oxidantes en la solución; sin embargo, en presencia de los antibióticos, la acumulación de oxidantes solo es apreciable cuando dichos contaminantes han desaparecido (luego de 4 minutos). Estos resultados sugieren que la eliminación de los fármacos ocurre de manera indirecta, vía reacción con las especies oxidantes electrogeneradas.

Con el fin de corroborar la anterior hipótesis, se realizó un experimento de control en el que se empleó sulfato de sodio, en lugar de cloruro de sodio, como electrolito soporte. Bajo dichas condiciones, contrario a lo observado en presencia de cloruro, la degradación de los contaminantes fue inferior al $5 \%$ en 5 min de tratamiento (datos no mostrados). Se ha reportado que los ánodos tipo DSA, como el Ti//rO $\mathrm{Ir}_{2}$, son excelentes catalizadores para la oxidación de cloruro $\left(\mathrm{Cl}^{-}\right)$a cloro $\left(\mathrm{Cl}_{2}\right)$; la hidrólisis de cloro permite la formación del ácido hipocloroso $(\mathrm{HOCl})$ y, finalmente, dicho ácido puede disociarse para producir el ion hipoclorito $\left(\mathrm{OCl}^{-}\right)$(ecuaciones 1 a 3 ) [13].

$$
\begin{aligned}
2 \mathrm{Cl}^{-} & \rightarrow \mathrm{Cl}_{2}+2 e^{-} \\
\mathrm{Cl}_{2}+\mathrm{H}_{2} \mathrm{O} & \rightarrow \mathrm{HOCl}+\mathrm{H}^{+}+\mathrm{Cl}^{-} \\
\mathrm{HOCl} & \rightarrow \mathrm{H}^{+}+\mathrm{ClO}^{-}
\end{aligned}
$$

Dichas especies reactivas de cloro, $\mathrm{Cl}_{2}, \mathrm{OCl}^{-} \mathrm{y}$ $\mathrm{HOCl}$ [20], tienen potenciales de reducción lo suficientemente altos ( $\mathrm{E}_{\text {red }}^{\mathrm{o}}$, volts): 1,30, 1,48 y 1,64, respectivamente [21], haciendo viable la oxidación de contaminantes orgánicos por estas especies [16]. Se ha reportado que las especies reactivas de cloro atacan sitios particulares como nitrógeno, azufre o sistemas aromáticos, mediante reacciones de oxidación, reacciones de adición a enlaces insaturados o reacciones de sustitución electrofílica a sitios nucleofílicos [22].

De lo anterior se puede deducir que la degradación electroquímica de los antibióticos bajo estudio es mediada por las especies de cloro electrogeneradas en la superficie del ánodo, las cuales pueden atacar preferencialmente el grupo amido, el grupo tioeter y el enlace carbono-cloro de las isoxazolilpenicilinas. Dichas reacciones ocurren de manera rápida, y menos de 5 minutos son suficientes para remover el contaminante.

\section{Seguimiento a la formación de los productos de degradación}

En la sección anterior se mostró cómo la electroquímica permite eliminar los antibióticos en estudio; sin embargo, el ácido hipocloroso ( $\mathrm{HOCl}$ ) es la especie de cloro activo (electrogenerado) predominante en el rango de $\mathrm{pH}$ estudiado (entre 6,0 y 7,0), que generalmente induce pequeñas modificaciones en el compuesto de origen, conduciendo a moléculas más oxidadas o cloradas [22]. Adicionalmente, a menudo los productos de degradación generados son menos susceptibles a la acción de las especies reactivas de cloro.

Con el objetivo de lograr una mayor comprensión del proceso, la figura 6 muestra los cromatogramas obtenidos a determinados intervalos de tiempo. En ellos es posible establecer la formación de 4 subproductos mayoritarios de degradación para la OXA con tiempos de retención a los 4,1 $\min (\mathrm{A}), 6,3 \mathrm{~min}(\mathrm{~B})$, 7,0 min (C) y 9,8 min (D), siendo los productos A y B los mayoritarios.

Para el caso de la CLX se detectaron 4 subproductos a los tiempos de retención: 4,9 (A), 7,7 (B), 11,3 (C) y 13,5 (D) minutos, siendo A y B los intermediarios mayoritarios, y finalmente, para la DCX se observaron, igualmente, 4 subproductos con tiempos de retención: 4,6 (A), 5,8 (B), 6,8 (C) y 10,6 (D) minutos, con los subproductos a los tiempos 6,8 (C) y 10,6 (D) minutos como los de mayor intensidad.

Un análisis de los cromatogramas obtenidos en el curso del tratamiento (datos no mostrados) indica que los subproductos generados para la OXA a los 4,1 min, para la CLX a los 4,8 min y para la DCX a los 6,8 minutos experimentan un incremento en la intensidad desde el primer minuto de tratamiento hasta los 5 minutos, momento en el cual empiezan a disminuir. A diferencia de ellos, los subproductos con tiempos cromatográficos de 6,3; 7,7 y 10,6 minutos para OXA, CLX y DCX, respectivamente, muestran un incremento desde 1 minuto y tienden a acumularse en la solución, mostrando que estos subproductos son más resistentes a la degradación. 


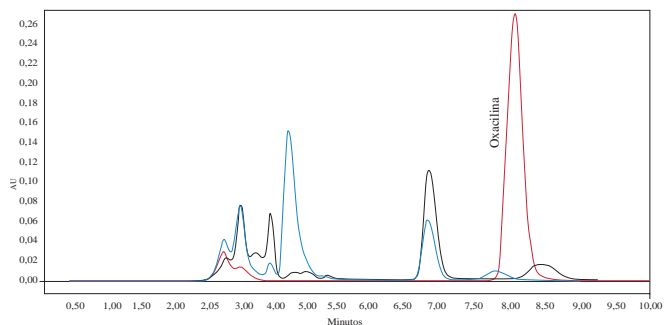

(a)

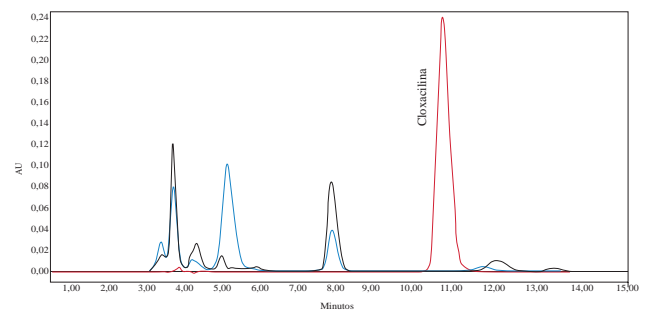

(b)

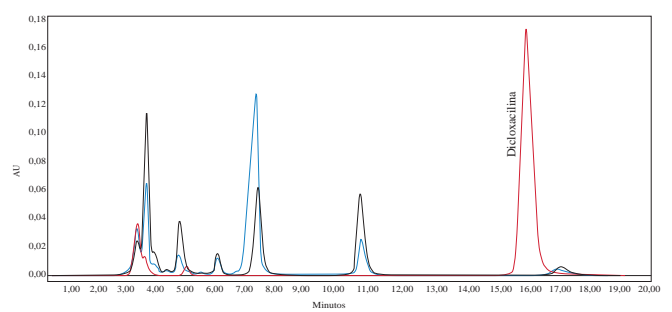

(c)

Figura 6. Cromatogramas indicando la formación de los productos de degradación (a) OXA, (b) CLX y (c) DCX después de 60 minutos de tratamiento. Líneas Roja: 0 minutos; Azul: 5 minutos; Negra: 60 minutos de tratamiento.

\section{Pérdida de la actividad antimicrobiana de las so- luciones tratadas}

En las secciones anteriores también se notó que algunos intermediarios orgánicos persisten al final del tratamiento; de esta manera, es relevante estudiar si la concentración remanente de los productos de degradación aún conserva alguna actividad antimicrobiana. El análisis de la actividad antimicrobiana usando bacterias que presenten una respuesta lineal con la concentración del agente bactericida debe ser realizado para establecer la sensibilidad del microorganismo a determinado antibiótico [23].

En el caso de los antibióticos $\beta$-lactámicos tipo isoxazolilpenicilinas, el Staphylococcus aureus es el microorganismo que presenta mayor susceptibilidad; por lo tanto, en este trabajo se utilizó una cepa de Staphylococcus aureus (ATCC 6538). Los resultados que evidenciaron la inhibición de la actividad de las soluciones de OXA, CLX y DCX antes, durante y después del tratamiento electroquímico, a intervalos de tiempo dados, son presentados en la figura 7. Como se observa en ella, la actividad antibacteriana de las muestras desaparece a los 5 minutos de tratamiento, lo que indicó que posterior a la desaparición del compuesto padre los productos de degradación producidos no presentan actividad antibiótica.

Ensayos realizados en ausencia de los antibióticos para evaluar el efecto antimicrobiano de los agentes oxidantes electrogenerados indicaron que, bajo las condiciones de trabajo, el microorganismo no es susceptible a las especies reactivas electrogeneradas $y$, por lo tanto, el agua obtenida después de realizar el tratamiento electroquímico no presenta actividad frente al Staphylococcus aureus, aun conteniendo especies de cloro activas. Dichos resultados indican que el tratamiento electroquímico es una técnica efectiva para la reducción del potencial antibiótico de aguas que presenten estos compuestos, disminuyendo el riesgo ambiental de la proliferación de bacterias resistentes a los antibióticos considerados en este estudio.

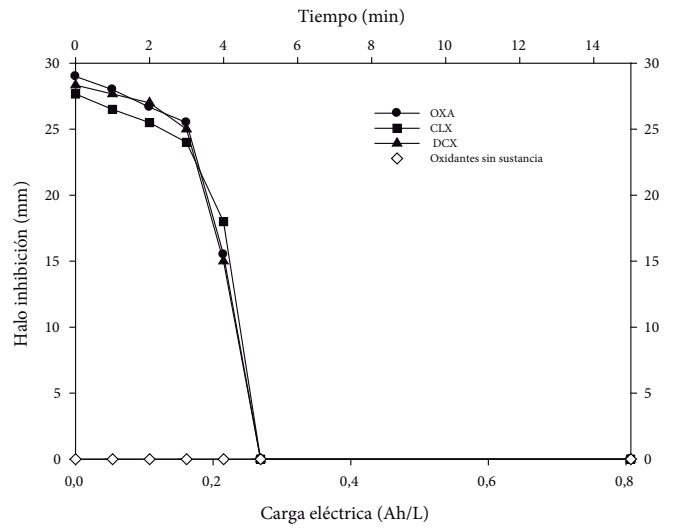

Figura 7. Evolución de la actividad antimicrobiana (halo de inhibición) durante tratamiento electroquímico de antibióticos $\beta$-lactámicos isoxazolilpenicilinas (203 $\left.\mu \mathrm{mol} \mathrm{L}{ }^{-1}\right) .121 \mathrm{~mA}, 0,225 \mathrm{~mol} \mathrm{~L}^{-1} \mathrm{NaCl}$ y 15 minutos de tratamiento.

\section{Conclusiones}

Este estudio mostró que la electroquímica, empleando un ánodo tipo DSA, como $\mathrm{Ti} / \mathrm{IrO}_{2}$, para la degradación de antibióticos tipo isoxazolilpenicilinas, es una alternativa muy prometedora en aguas que contienen niveles apreciables de iones cloruro. 
La evaluación del desempeño cinético del sistema, tomando la CLX como sustancia modelo, mostró que la corriente aplicada, la concentración del electrolito soporte y la interacción entre estos dos parámetros fueron las variables que ejercieron un impacto significativo sobre la eficiencia del proceso electroquímico. La concentración del antibiótico, por su parte, no afectó la eficiencia del tratamiento. De otro lado, el análisis de la acumulación de las especies oxidantes y su interacción con los antibióticos indicó que la vía indirecta es la ruta degradatíva predominante; siendo el HOCl la principal especie responsable de la degradación de estos compuestos. De manera interesante, la evolución de los intermediarios evidenció que algunos productos de degradación generados a tiempos cortos del tratamiento fueron también degradados por el sistema electroquímico. Adicionalmente, la remoción de la actividad antimicrobiana, una vez se degrada el $100 \%$ de las isoxazolilpenicilinas, sugiere que los intermediarios remanentes de la oxidación no presentan actividad antibiótica, y que la oxidación electroquímica induce cambios estructurales en las moléculas de los antibióticos tales que su actividad biológica se elimina.

\section{Agradecimientos}

A Colciencias, por el apoyo financiero a este trabajo a través del proyecto: "Implementación de metodologías eficientes y confiables para degradar residuos de antimicrobianos en el hogar y en efluentes industriales" Código: 1115-569-33692 (RC 00072013); y al Fondo Nacional Suizo (Swiss National Foundation) por su ayuda económica mediante el proyecto "Treatment of the hospital wastewaters in Cote d'Ivoire and in Colombia by advanced oxidation processes".

\section{Referencias}

[1] M. R. Periago, "La resistencia a los antimicrobianos: un factor de riesgo para las enfermedades infecciosas", Revista Panamericana de Salud Pública, vol. 30, pp. 507-509, 2011.

[2] A. Junza, N. Dorival-García, A. Zafra-Gómez, D. Barrón, O. Ballesteros, J. Barbosa et al., "Multiclass method for the determination of quinolones and $\beta$-lactams, in raw cow milk using dispersive liquid-liquid microextraction and ultra high performance liquid chromatographytandem mass spectrometry", Journal of Chromatography A, vol. 1356, pp. 10-22, 2014.

[3] F. García, "Resistencia bacteriana a antibióticos”, Acta Médica Costarricense, vol. 43, pp. 101-102, 2001.

[4] M. A. Guzmán M., J. Salinas L., P. Toche P., and A. Afani S, "Alergia a b-lactámicos", Revista chilena de infectología, vol. 21, pp. 285-298, 2004.

[5] P. S. M. Dunlop, M. Ciavola, L. Rizzo, D. A. McDowell, and J. A. Byrne, "Effect of photocatalysis on the transfer of antibiotic resistance genes in urban wastewater". Catalysis Today.

[6] OMS, Resistencia a los antimicrobianos, 2013.

[7] Y. Ishii, C. Ueda, Y. Kouyama, K. Tateda, and K. Yamaguchi, "Evaluation of antimicrobial susceptibility for $\beta$-lactams against clinical isolates from 51 medical centers in Japan (2008)", Diagnostic Microbiology and Infectious Disease, vol. 69, pp. 443-448, 2011.

[8] J. M. Cha, S. Yang, and K. H. Carlson, "Trace determination of $\beta$-lactam antibiotics in surface water and urban wastewater using liquid chromatography combined with electrospray tandem mass spectrometry", Journal of Chromatography A, vol. 1115, pp. 46-57, 2006.

[9] M. E. Epstein, M. Amodio-Groton, and N. S. Sadick, "Antimicrobial agents for the dermatologist. I. $\beta$-Lactam antibiotics and related compounds", Journal of the American Academy of Dermatology, vol. 37, pp. 149-165, 1997.

[10] Today's Drugs: Penicillins And Cephalosporins, Today's Drugs: Penicillins And Cephalosporins, The British Medical Journal, vol. 2, no. 5604, pp. 542-545, 1968. Retrieved from http://www.jstor.org/stable/20392812

[11] M. A. Oturan and E. Brillas, "Electrochemical Advanced Oxidation Processes (EAOPs) for Environmental Applications", Portugaliae Electrochimica Acta, vol. 25, pp. 1-18, 2007. 
[12] M. Panizza, A. Barbucci, R. Ricotti, and G. Cerisola, "Electrochemical degradation of methylene blue", Separation and Purification Technology, vol. 54, pp. 382-387, 2007.

[13] M. Panizza and C. A. Martinez-Huitle, "Role of electrode materials for the anodic oxidation of a real landfill leachate - Comparison between Ti$\mathrm{Ru}-\mathrm{Sn}$ ternary oxide, $\mathrm{PbO} 2$ and boron-doped diamond anode", Chemosphere, vol. 90, pp. 1455-1460, 2013.

[14] R. E. Palma-Goyes, F. L. Guzmán-Duque, G. Peñuela, I. González, J. L. Nava, and R. A. Torres-Palma, "Electrochemical degradation of crystal violet with BDD electrodes: Effect of electrochemical parameters and identification of organic by-products", Chemosphere, vol. 81, pp. 26-32, 2010.

[15] R. A. Torres, W. Torres, P. Peringer, and C. Pulgarin, "Electrochemical degradation of $\mathrm{p}$ substituted phenols of industrial interest on $\mathrm{Pt}$ electrodes: Attempt of a structure-reactivity relationship assessment", Chemosphere, vol. 50, pp. 97-104, 2003.

[16] R. A. Torres, V. Sarria, W. Torres, P. Peringer, and C. Pulgarin, "Electrochemical treatment of industrial wastewater containing 5amino-6-methyl-2-benzimidazolone: toward an electrochemical-biological coupling", Water Research, vol. 37, pp. 3118-3124, 2003.

[17] U. S. Pharmacopeial, "USP 35/NF 30 The United States Pharmacopeia”, p. 4141, 2012.
[18] D. C. Montgomery and G. C. Runger, "Applied Statics and Probability for Engineer. Design and Analysis of Experiments", 3 ed., John Willey \& Sons, INC., 2003.

[19] R.A. Torres, R. Mosteo, C. Pétrier, C. Pulgarín. "Experimental design approach to the optimization of ultrasonic degradation of alachlor and enhancement of treated water biodegradability". Ultrasonics Sonochemistry, vol. 16, no. 3, 425-430, 2009.

[20] T. González, J. R. Domínguez, P. Palo, J. Sánchez-Martín, and E. M. Cuerda-Correa, "Development and optimization of the BDDelectrochemical oxidation of the antibiotic trimethoprim in aqueous solution", Desalination, vol. 280, pp. 197-202, 2011.

[21] P. D. Philip C. Singer and P. D. David A. Reckhow, "Chemical Oxidation", in Water quality and treatment: a handbook of community water supplies, McGraw-Hill, Fifth Ed., p. 1248, 1999.

[22] M. Deborde and U. von Gunten, "Reactions of chlorine with inorganic and organic compounds during water treatment-Kinetics and mechanisms: A critical review", Water Research, vol. 42, pp. 13-51, 2008.

[23] C. Reyes, J. Fernández, J. Freer, M. A. Mondaca, C. Zaror, S. Malato, et al., "Degradation and inactivation of tetracycline by $\mathrm{TiO} 2$ photocatalysis", Journal of Photochemistry and Photobiology A: Chemistry, vol. 184, pp. 141-146, 2006. 